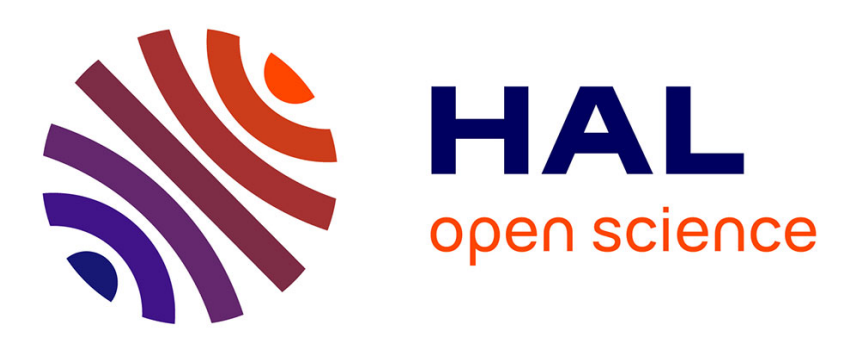

\title{
Abnormal K -point dependent electromagnetic beam propagation in a graphenelike triangular metallic photonic structure
}

Kang Wang

\section{- To cite this version:}

Kang Wang. Abnormal K -point dependent electromagnetic beam propagation in a graphenelike triangular metallic photonic structure. Physical Review B: Condensed Matter and Materials Physics (1998-2015), 2019, 100 (11), pp.115140. 10.1103/PhysRevB.100.115140 . hal-02322839

\section{HAL Id: hal-02322839 \\ https://hal.science/hal-02322839}

Submitted on 2 Sep 2021

HAL is a multi-disciplinary open access archive for the deposit and dissemination of scientific research documents, whether they are published or not. The documents may come from teaching and research institutions in France or abroad, or from public or private research centers.
L'archive ouverte pluridisciplinaire HAL, est destinée au dépôt et à la diffusion de documents scientifiques de niveau recherche, publiés ou non, émanant des établissements d'enseignement et de recherche français ou étrangers, des laboratoires publics ou privés. 


\title{
Abnormal $K$-point dependent electromagnetic beam propagation in a graphenelike triangular metallic photonic structure
}

\author{
Kang Wang (1) \\ Laboratoire de Physique des Solides, UMR CNRS/Université Paris-Sud, Université Paris-Saclay, 91405 Orsay, France
}

(Received 17 July 2019; revised manuscript received 29 August 2019; published 18 September 2019)

\begin{abstract}
We study the electromagnetic wave propagation in a graphenelike triangular metallic photonic structure and show that, associated to trigonally warped Dirac cones, the transport properties resulting from an incident beam at the armchair edge display drastically different behaviors as compared to the results obtained for other analogous systems. Namely, at $K$ point, only the center beam associated to the lower Dirac cone and the side beams to the upper cone and, at $K^{\prime}$ point, only the center beam associated to the lower cone display significant intensities. The other expected beams are all strongly inhibited. This can be related to the metallic nature of the structure, where the low frequency electromagnetic bands are formed by local resonance modes confined inside the structural units. A propagating wave should follow the structure local patterns that govern its field distribution, whose symmetry properties determine its excitation by an incident wave.
\end{abstract}

DOI: 10.1103/PhysRevB.100.115140

\section{INTRODUCTION}

Graphenelike photonic structures provide opportunities for optically probing graphene-related physical properties and attract enormous interest. Many fundamental phenomena, including conical diffraction, gap soliton, pseudodiffusive transmission, quantum Hall-like effect, zero refractive index, and valley-polarized beam propagation [1-5] have been studied. Indeed, the spectrum modifications of an electromagnetic (EM) emission, resulting from the interaction with such photonic structures, provide information on the dispersion properties of the Dirac cones as well as their coupling with the incident wave. Potential applications, such as valley photonics exploring optically the valley degree of freedom, analogous to valleytronics [6], have recently been proposed for information processing [7].

Various dielectric photonic structures, including triangular [2,3,8], honeycomb [1,2], and square [4] ones, can create Dirac cones, while, regarding the metallic structures, Dirac cones can be formed in triangular $[9,10]$ and isogonal hexagonal [11] ones. In fact, for a metallic lattice, the low frequency EM field follows the dual lattice distribution, with cavitylike resonance modes formed inside the structural units [12]. For a triangular metallic structure with high filling rate, the EM field follows a honeycomb distribution [10], and such a structure is equivalent to a honeycomb atomic one.

It is known that, in a honeycomb structure, the Dirac cones undergo trigonal warping for frequencies departing from the Dirac level [13]. When the isofrequency contours of the Dirac cones become nearly triangular, beam splitting and self-collimation associated to different $K$ points occur due to the anisotropic group velocity distribution [5], offering possibilities for wave propagation control and beam manipulation. Studies on $K$-point dependent EM wave propagation in graphenelike photonic structures are limited to dielectric

*kang.wang@u-psud.fr structures [5,14], where the Dirac cone formation can be described by nearly free photon approximation using the plane wave expansion method [15]. These studies concern, however, only the lower cones. Concerning the upper Dirac cones, there are reports on analogous situations of electron beam propagation in mono- and bilayer graphenes [16,17].

To our knowledge, there is until now no investigation on $K$ point dependent EM beam propagations in metallic photonic structures. Metallic structures possess their specific properties and interests. Generally of low fabrication costs, they need only a few number of periods to achieve band gaps [18], allowing smaller sample sizes in practice. More particularly, due to their surface screening, the metallic elements exclude the EM field, producing cavitylike local resonance modes confined inside the structural units delimited by these elements [12]. Low-frequency band formation in metallic structures can be described by a tight binding (TB) model, with the crystal eigenmodes as superpositions of these local modes [10-12]. As a consequence, EM wave propagation in such structures is realized through the coupling between the structural units, and will be determined by the structure local patterns by following the structural unit distribution.

We investigate in the present work the propagation of EM waves associated to the Dirac cones from the armchair edge in a triangular metallic photonic structure. We show that, compared to the studies mentioned above $[5,14,16]$, the beam propagation in such a photonic structure displays drastically different behaviors.

\section{ELECTROMAGNETIC BEAM PROPAGATION}

\section{A. Band symmetry and Dirac cone}

A triangular lattice [Fig. 1(a)] belongs to the symmetry group $D_{6 h}$ and contains two families (A and B) of triangles as structural units. The metallic structure is constructed by infinite cylinders, with radius $r=0.25 a$ ( $a$ being the lattice constant) and dielectric constant set to negative infinity, placed 

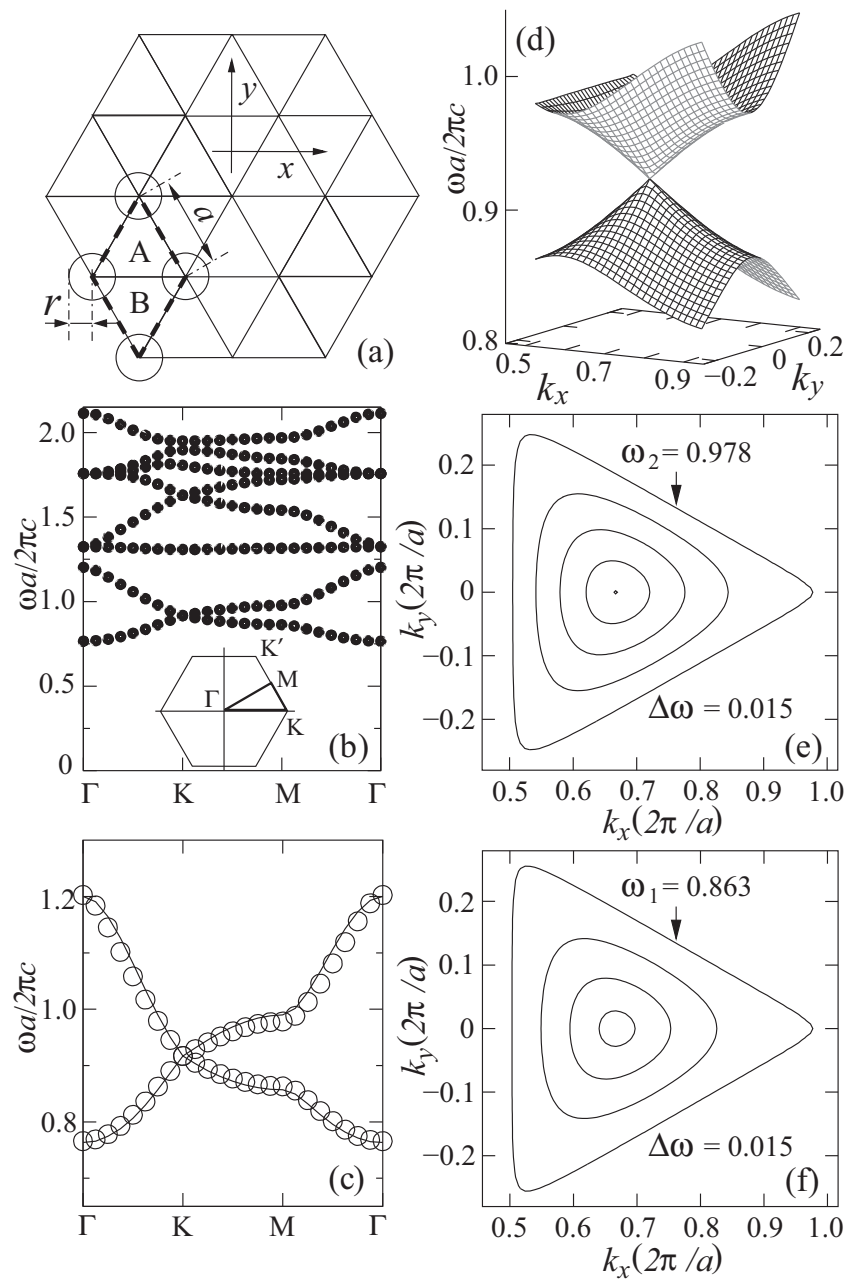

FIG. 1. Metallic triangular photonic structure (a) and the corresponding EM frequency band diagram (b). Dirac cones are formed by the first two bands (c). A 3D view of the Dirac cone at $K(4 \pi / 3 a, 0)$ is shown in (d). Isofrequency contours of the upper and lower cone at the same $K$ point are depicted in (e) and (f), where the frequency level $\left(\omega_{1}\right.$ and $\left.\omega_{2}\right)$ as well as the contour interval $(\Delta \omega)$ values are normalized to $\omega a / 2 \pi c$.

at the lattice nodes in an air background. The EM frequency band diagram for TM polarization (the electric field parallel to $z$ axis), obtained by solving Maxwell's equations using the finite-difference time-domain (FDTD) method, is displayed in Fig. 1(b). Dirac cones are formed by the first two bands replotted in Fig. 1(c) [10]. These two bands are delimited by two gaps: the plasma gap [19] from $0.766(\omega a / 2 \pi c)$ down to zero frequency and a band gap from 1.203 to $1.308(\omega a / 2 \pi c)$ separating them from the third band. An EM wave in the frequency range of these two bands propagating in the structure will not encounter other bands. A 3D view of the Dirac cone at $K(4 \pi / 3 a, 0)$ point is shown in Fig. 1(d). The upper and lower Dirac cones at the $K$ point are depicted by isofrequency contours in the same figure [1(e) and 1(f)]. Indeed, departing from the Dirac frequency $\omega_{D} \approx 0.917$, the isofrequency lines undergo trigonal distortion and take quasitriangular forms at $\omega_{1} \approx 0.863$ and $\omega_{2} \approx 0.978(\omega a / 2 \pi c)$ for respectively the lower and the upper cones.

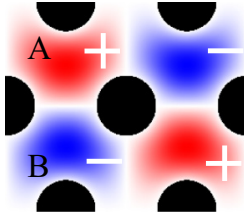

(a)

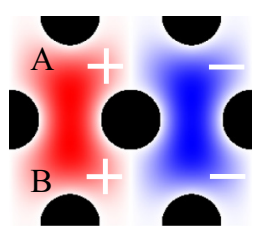

(b)

(c)

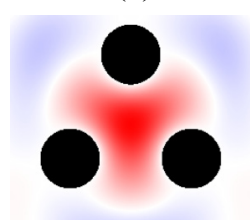

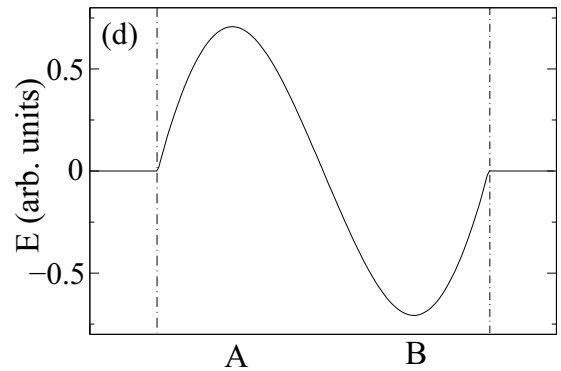

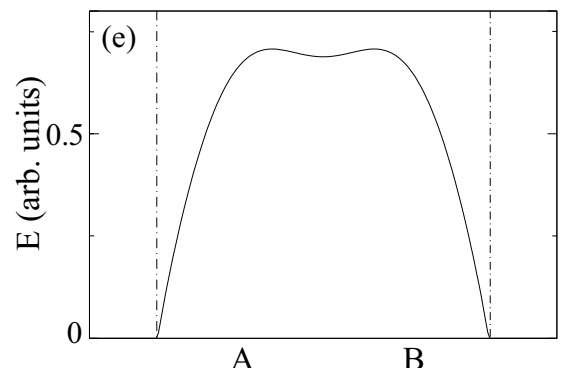

FIG. 2. Electric field distributions corresponding to the upper (a) and lower (b) Dirac band branch at $k=(\pi / a, 0)$, and in an individual triangle (c). The corresponding field magnitudes along a slice passing by the A and B triangle centers are shown in (d) and (e). The \pm signs indicate the field polarities.

The band formation can be described by a TB model [10] [solid lines in Fig. 1(c)]. Recall that, in a such model, the crystal wave function is expressed as the sum of local wave functions, $\left|\phi_{A}\right\rangle$ and $\left|\phi_{B}\right\rangle$, corresponding to the electric field distributions of the resonance modes formed inside $\mathrm{A}$ and B triangles. Along $\Gamma-K$ direction, the modes $\left|\phi_{A}\right\rangle$ and $\left|\phi_{B}\right\rangle$ contribute equally, in absolute value, to the crystal wave function:

$$
\begin{aligned}
\left|\Psi_{\mathbf{k}}(\mathbf{r})\right\rangle= & \sum_{m} \frac{e^{i \mathbf{k} \mathbf{R}_{m}}}{\sqrt{2 N}}\left[\left|\phi_{A}\left(\mathbf{r}-\mathbf{R}_{m}-\mathbf{d}_{A}\right)\right\rangle\right. \\
& \left. \pm\left|\phi_{B}\left(\mathbf{r}-\mathbf{R}_{m}-\mathbf{d}_{B}\right)\right\rangle\right]
\end{aligned}
$$

with $\sum_{m}$ summing over the unit cells. The wave function is characterized by a mirror or a mirror inversion symmetry with respect to the plane defined by the $\Gamma-K$ line and the $z$ axis, described by the \pm signs and corresponding to the lower or upper Dirac band branches.

The symmetry relation can further be checked by the field distributions. As an example, the electric field distributions of the two band branches at $k=(\pi / a, 0)$, together with that in an individual triangle, obtained by the FDTD method, are displayed in Fig. 2. This figure shows that, for the lower or upper band, the electric fields in the A and B triangles display the same or opposite polarities. Electric field magnitudes, obtained by the same method, along a slice passing by the $\mathrm{A}$ and $\mathrm{B}$ triangle centers and with the maximum total intensities normalized to unity for each band, are displayed in the same figure. This confirms the mirror and mirror inversion symmetries.

The above analyses can be described by the group theory. At $\Gamma$ point, the $D_{6 h}$ group has two irreducible representations $A_{1 g}$ and $B_{1 u}$ [20], characterized, respectively, by a mirror 
and a mirror inversion symmetry with respect to the plane defined by a $\Gamma-K$ line and the $z$ axis, that become $A_{1}$ and $B_{1}$ representations of the $C_{2 v}$ group [20] along $\Gamma-K$. The crystal wave function can be described by $A_{1}$ and $B_{1}$.

This will impact directly the wave propagation for an incident beam along the $\Gamma-K$ direction, where self-collimation effects are obtained for EM waves and electron beams in respectively dielectric graphenelike photonic structures and atomic graphenes, with, perpendicular to the isofrequency contours, one self-collimated center beam along the incident direction and two side beams with weaker intensity along the $\pm \pi / 3$ directions, associated to the lower or upper Dirac cones $[5,14,16]$. In the present case, a center beam described by the $A_{1}$ representation can be coupled to a TM polarized incident beam along the $\Gamma-K$ direction, while there cannot be coupling for a center beam described by the $B_{1}$ representation, the incident beam being characterized by a mirror symmetry plane defined by the $\Gamma-K$ line and the $z$ axis. Similarly, the coupling with the side beams will also be determined by their symmetry relations with the field patterns associated to the incident beam at the sample edge.

\section{B. Electromagnetic beam propagation from the armchair edge}

Let us consider a sample containing $18 \times 15$ periods (Figs. 3 and 4). The TM polarized beams are injected on the armchair edge through a metallic waveguide limiting the

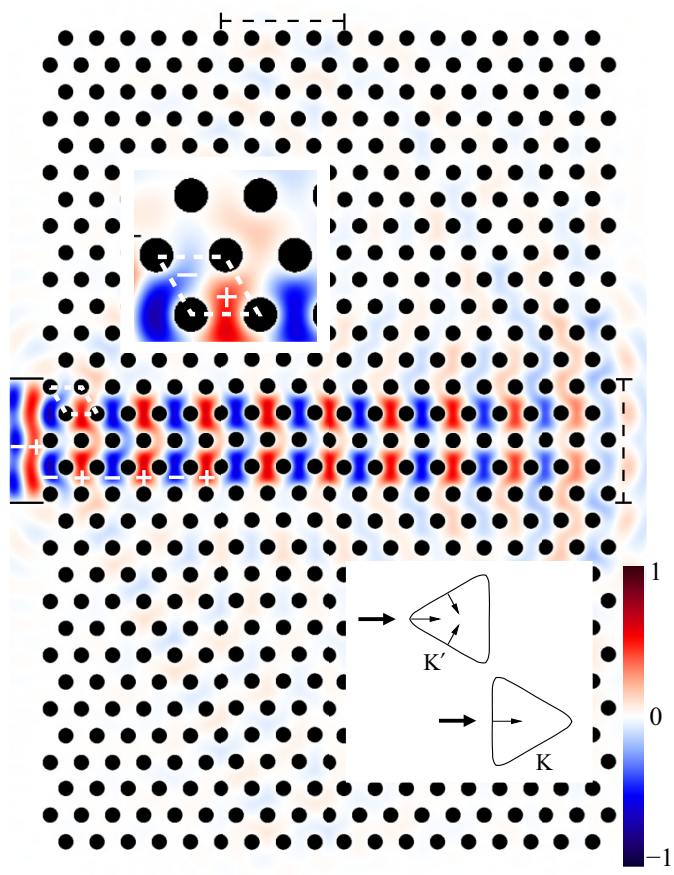

FIG. 3. Electric field distribution of a monochromatic wave of frequency $\omega_{1}$, propagating in an $18 \times 15$ period sample. The incident beam is injected through a wave guide at the left side. The inset in the lower part indicates the incident (thick arrows) and expected propagation (thin arrows) directions. The inset in the upper part shows a magnified zone around the unit cell in the incident region. The \pm signs indicate the field polarities and dashed lines the regions where transmissions are considered.

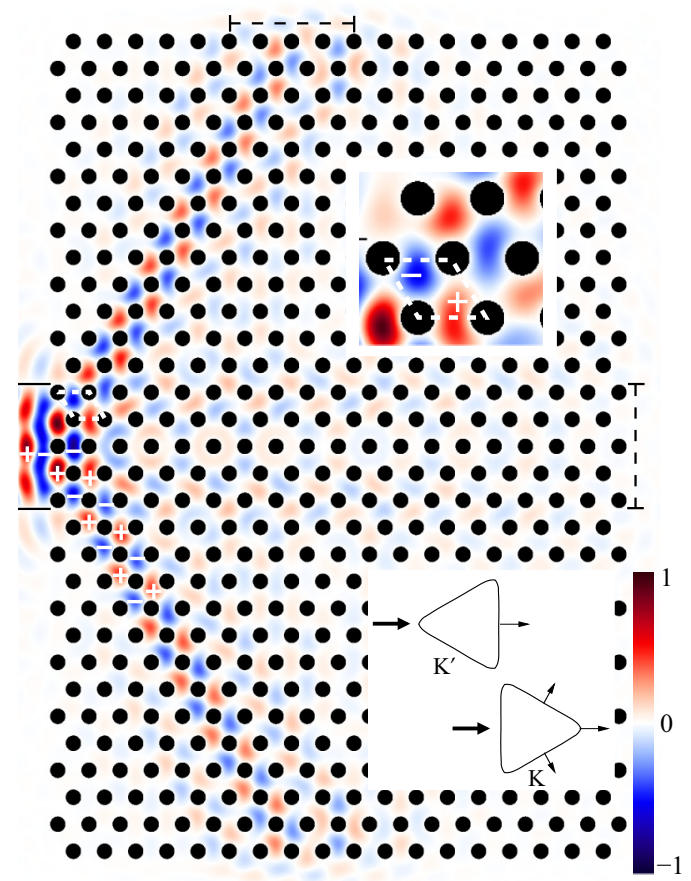

FIG. 4. Same configuration as in Fig. 3, but for incident wave frequency $\omega_{2}$.

incident beam width to $4 a$. Two monochromatic waves, with $\omega_{1}=0.863$ and $\omega_{2}=0.978(\omega a / 2 \pi c)$, corresponding to the lower and upper nearly triangular isofrequency contour levels of the Dirac cones (see Fig. 1), are considered. The wave propagation in the sample is obtained using the FDTD method, and images of the electric field distributions are displayed in the same figures. It is obvious that, compared to the lower part insets in these figures, at $\omega_{1}$, the expected side beams associated to the $K^{\prime}$ point are almost absent, and only the center beam associated to $K$ and $K^{\prime}$ points displays significant amplitude, while, at $\omega_{2}$, the expected center beam associated to $K$ and $K^{\prime}$ points is almost absent, and only the two side beams associated to the $K$ point display significant amplitude.

For a more quantitative description of the propagation behaviors, we consider the transmission spectra, normalized by the incident flux, in two outgoing regions with the same width as the incident waveguide. One, for the center beam direction, is placed across the sample in front of the incident beam; the other, for one of the side beam directions, is placed on the sample upper side at $\pi / 3$ angle (see the dashed lines in Figs. 3 and 4). The transmission spectra obtained by the FDTD method, centered at Dirac frequency $\omega_{D}$, are displayed in Fig. 5, with the transmission spectrum in the side beam direction multiplied by 2 .

Figure 5 shows that, in the center beam direction, the transmission associated to the lower cone is predominant, and reaches its maximum near $\omega_{1}$, corresponding to the selfcollimation effect at $K$ and $K^{\prime}$ points, while, in the side beam directions, the transmission associated to the upper cone is predominant, and reaches its maximum near $\omega_{2}$, corresponding to the beam splitting effect at the $K$ point. The two maximum transmission peaks have comparable magnitudes. The transmissions in the side beam directions associated to 


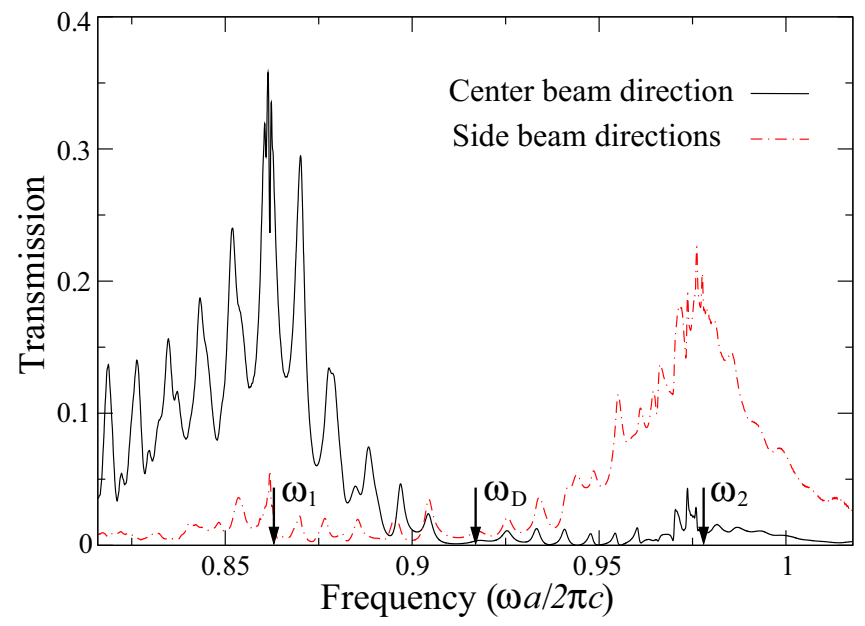

FIG. 5. Transmission spectra in the center and side beam directions in the vicinity of the Dirac frequency $\omega_{D}$. The incidence and transmission regions are depicted in Figs. 3 and 4. The frequency levels $\omega_{1}$ and $\omega_{2}$ are also indicated.

the lower cone and in the center beam direction associated to the upper cone are much weaker. Their maxima, around $\omega_{1}$ (the side beams at the $K^{\prime}$ point) and $\omega_{2}$ (the center beam at $K$ and $K^{\prime}$ points), are one order of magnitude lower than their counterparts. This corroborates the field distributions in Figs. 3 and 4.

As mentioned in Sec. II A, these particular behaviors are to be linked to the symmetries of both the incident beam and the frequency bands. Figures 3 and 4 show that the field distribution of the center beam at $\omega_{1}$ can be described by the $A_{1}$ representation, with a mirror symmetry with respect to the $x z$ plane, while, at $\omega_{2}$, the field distribution of each of the side beams can be described by the $B_{1}$ representation, with a mirror inversion symmetry with respect to the plane defined by the $z$ axis and the propagation direction. This is in agreement with the band symmetry below and above the Dirac frequency level discussed in Sec. II A. Moreover, at $\omega_{2}$, the field distribution of the two side beams together are described by a mirror symmetry with respect to the $x z$ plane. So the symmetries of these propagating beams are compatible with that of the incident beam and can be coupled to the latter. The center beam at $\omega_{2}$, that should also belong to the $B_{1}$ representation, cannot be coupled to the incident beam. The weak transmission in this direction can be attributed to mode excitations at the edges of the incident beam.

Contrary to the pair of side beams at $\omega_{2}$, the two expected side beams at $\omega_{1}$, that should be described each by the $A_{1}$ representation, display very weak transmission. Indeed, for both the lower and upper cones, the two pairs of expected side beams (two $A_{1}$ and two $B_{1}$ beams) are both symmetrically compatible with the incident beam. This condition is, how- ever, not enough, and the symmetry relations of each beam with the incident field patterns should also be considered. As a matter of fact, for the side beams associated to the lower cone, considering a unit cell at the sample edge at the beginning of the paths in the $\pi / 3$ direction (see the upper part inset in Fig. 3), the local electric fields in the two triangles forming the unit cell, though with different magnitudes, are of opposite polarities, due to the alternative mode polarities in the center beam direction. The coupling with a beam propagating along the $\pi / 3$ direction and described by the $A_{1}$ representation is destructive. This accounts for the strong inhibition of the side beams at $\omega_{1}$. On the contrary, the local electric field in the same triangles, associated to the upper cone (the upper part inset in Fig. 4), can be constructively coupled with a beam described by $B_{1}$ and propagating in the same direction. This explains the side beam excitation at $\omega_{2}$.

From a functional point of view, as compared to the previous investigations in analogous systems [5,14,16,17], a triangular metallic photonic structure allows, on one hand, a better beam self-collimation associated to the lower Dirac cone, the side beams at the $K^{\prime}$ point being almost absent, and, on the other hand, associated to the upper cone, it enables beam splitting at one Dirac point by allowing two strong side beams and inhibiting the center one, with the exclusion of the other Dirac point, allowing addressing directly and separately each Dirac point by varying the edge and angle of incidence, and obtaining the response from a selected single Dirac point. Further, compared to the zigzag edge, the beam splitting at the armchair edge is characterized by a splitting angle twice as large.

\section{CONCLUSION}

In summary, due to the metallic nature of the structure that imposes local EM resonance mode distributions for the frequency bands, the EM wave propagation associated to the trigonally warped Dirac cones in a graphenelike triangular metallic photonic structure, excited by an incident beam at the armchair edge, displays drastically different behaviors as compared to other analogous systems. Indeed, the incident beam is collimated at $K$ and $K^{\prime}$ points, associated to the lower Dirac cones, while, associated to the upper cones, it is split into two beams with a $2 \pi / 3$ angle at the $K$ point. The other beam excitations expected for nearly triangular isofrequency contours and obtained previously for analogous systems are all strongly inhibited. This highlights the singularity of the graphenelike metallic photonic structure in its interaction with the EM waves, and may find application in valley photonics in the designs of a high efficiency beam collimator to convey information associated to both valleys, and large angle splitter for the beam carrying information associated to a single specific valley.
[1] O. Peleg, G. Bartal, B. Freedman, O. Manela, M. Segev, and D. N. Christodoulides, Phys. Rev. Lett. 98, 103901 (2007).
[2] R. A. Sepkhanov, Y. B. Bazaliy, and C. W. J. Beenakker, Phys Rev. A 75, 063813 (2007). 
[3] S. Raghu and F. D. M. Haldane, Phys. Rev. A 78, 033834 (2008).

[4] X. Huang, Y. Lai, Z. H. Hang, H. Zheng, and C. T. Chan, Nat. Mater. 10, 582 (2011).

[5] J. L. Garcia-Pomar, A. Cortijo, and M. Nieto-Vesperinas, Phys. Rev. Lett. 100, 236801 (2008).

[6] A. Rycerz, J. Tworzydlo, and C. W. J. Beenakker, Nat. Phys. 3, 172 (2007).

[7] J.-W. Dong, X.-D. Chen, H. Zhu, Y. Wang, and X. Zhang, Nat. Mater. 16, 298 (2017).

[8] M. Plihal and A. A. Maradudin, Phys. Rev. B 44, 8565 (1991).

[9] S. Bittner, B. Dietz, M. Miski-Oglu, P. Oria Iriarte, A. Richter, and F. Schäfer, Phys. Rev. B 82, 014301 (2010).

[10] K. Wang, Mater. Res. Express 3, 115007 (2016).

[11] K. Wang, Phys. Rev. B 97, 125146 (2018).
[12] K. Wang, J. Opt. Soc. Am. B 31, 1273 (2014).

[13] R. Saito, G. Dresselhaus, and M. S. Dresselhaus, Phys. Rev. B 61, 2981 (2000).

[14] F. Deng, Y. Sun, X. Wang, R. Xue, Y. Li, H. Jiang, Y. Shi, K. Chang, and H. Chen, Opt. Express 22, 23605 (2014).

[15] F. D. M. Haldane and S. Raghu, Phys. Rev. Lett. 100, 013904 (2008).

[16] Z. Wang and F. Liu, ACS Nano 4, 2459 (2010).

[17] C. Park, J. Appl. Phys. 118, 244301 (2015).

[18] M. M. Sigalas, C. T. Chan, K. M. Ho, and C. M. Soukoulis, Phys. Rev. B 52, 11744 (1995).

[19] See, for example, J. B. Pendry, A. J. Holden, D. J. Robbins, and W. J. Stewart, J. Phys.: Condens. Matter 10, 4785 (1998).

[20] See, for example, J. R. Ferraro and K. Nakamoto, Introductory Raman Spectroscopy (Academic Press, New York, 1994), pp. 310 and 337. 